\title{
R\&D of a SIMPLE bubble chamber for dark matter searches
}

\author{
M. Felizardo a , T.A. Girard, I. Lazaro, A.C. Fernandes, T. Morlat, J.G. Marques, \\ and A. Kling \\ $\mathrm{C}^{2} \mathrm{TN}$, Centro de Ciências e Tecnologias Nucleares, Instituto Superior Técnico, Universidade de \\ Lisboa, E.N. 10 ao km 139,7, 2695-066 Bobadela LRS, Portugal
}

\begin{abstract}
SIMPLE (Superheated Instrument for Massive ParticLe Experiments) intends the realization and full field tests of a $25 \mathrm{~kg}$, low background, low frequency-instrumented $\mathrm{BC}$ (Bubble Chamber), with data from the $\mathrm{BC}$ testing and a small array demonstration providing new physics results. We report on the development of a $1 \mathrm{~kg}$ freon $\mathrm{BC}$ prototype, including chamber recompression system design and testing with initial acoustic detection of bubble formation. Realization of full field tests leading to a $25 \mathrm{~kg}$, overall $10^{-6}$ recoil evt $/ \mathrm{kgF} / \mathrm{d}$ background, low frequencyinstrumented $\mathrm{BC}$, incorporating several innovations designed, are intended to serve for an anticipated $2500 \mathrm{kgd}$ exposure, resulting in new exclusion contours with sensitivities of $2 \times 10^{-8}$ and $5 \times 10^{-5} \mathrm{pb}$ at $35 \mathrm{GeV}$ in the SI and SD sector of the WIMP phase space, respectively.
\end{abstract}

\section{Introduction}

The direct detection of astroparticle dark matter has returned to the spotlight of forefront physics. Its existence is now well-established by indirect means, to include a wealth of observational data but, despite the progress made in the direct search effort, over 10 orders of magnitude in sensitivity since 1984, no candidates have appeared in searches covering a range from about $10 \mathrm{GeV}$ to $1 \mathrm{TeV}$. Such astroparticle candidates are the leading candidates for dark matter particles, fitting nicely into the standard model of particle physics. Among the several technologies in current use is the bubble chamber (BC), based on the phase transition of a superheated liquid to its gas phase by energy deposition in the liquid, which must fulfil two thermodynamic conditions, which together render the detector effectively insensitive $>10^{10}$ to the majority of traditional particle backgrounds ( $\gamma$ 's, $\beta$ 's, cosmic $\mu$ 's) which plague the other more conventional search detectors. Additional advantages over conventional techniques include a relative inexpensiveness, a relative high efficiency in effective/raw exposure ( $\sim 80 \%$ vs. $40-50 \%)$, a simpler data acquisition/analyses, an ability to achieve low recoil energies, and reduction of the possible WIMP phase space via easy substitution of different superheated liquids. In short, the technique ranks among the most competitive dark matter search approaches. A BC requires a recompression system: this is based on a simple piston configuration to prevent $\mathrm{N}_{2}$ recompression gas from refrigerant/gel contact because of solubility, and for robustness. Its basic response to a nucleation event is a sensed pressure increase which provokes an immediate piston drop to increase the pressure to 10 bar (at which the gas is recompressed to a metastable liquid state), followed by a pressure

\footnotetext{
${ }^{a}$ Corresponding author: felizardo@ctn.tecnico.ulisboa.pt
}

(C) The Authors, published by EDP Sciences. This is an Open Access article distributed under the terms of the Creative Commons Attribution License 4.0 (http://creativecommons.org/licenses/by/4.0/). 
decrease to the operational pressure for the superheated state ( 2 bar in the case of $\mathrm{C}_{2} \mathrm{ClF}_{5}$ ); the entire recompression cycle requires $\sim 200 \mathrm{~s}$. Any acoustic signal related to gas bubble nucleation and growth in the liquid halocarbon must appear in coincidence with the pressure increase resulting from the same process. The shock wave produced by the bubble nucleation and expansion can be recorded by an acoustic sensor and converted into an electrical pulse. This pulse tends to be a damped sinusoid with a typical duration of several milliseconds. The projected $\mathrm{BC}$ sensitivity for dark matter searches demands an intrinsic background contribution of $\leq 10^{-6}$ events $/ \mathrm{kgF} / \mathrm{d}$.

\section{Past SIMPLE studies and field tests}

Phase II of SIMPLE searched for astroparticle dark matter using superheated liquid $\mathrm{C}_{2} \mathrm{ClF}_{5}$ droplet detectors. Each droplet generally required an energy deposition with linear energy transfer $(\mathrm{LET}) \geq 150 \mathrm{keV} / \mu \mathrm{m}$ for a liquid-to-gas phase transition, providing an intrinsic rejection against minimum ionizing particles of order $10^{-10}$, and reducing the backgrounds to primarily $\alpha$ and neutron-induced recoil events. The droplet phase transition generated a millimetric-sized gas bubble that was recorded by acoustic means. We described the SIMPLE detectors, their acoustic instrumentation, and the characterizations, signal analysis and data selection, which yielded a particle-induced, "true nucleation" event detection efficiency of better than $97 \%$ at a $95 \%$ C.L. The recoil- $\alpha$ event discrimination, determined using detectors first irradiated with neutrons and then doped with alpha emitters, provided a recoil identification of better than $99 \%$. The science measurements, comprising two shielded arrays of fifteen detectors each and a total exposure of $18.24 \mathrm{kgd}$, were thoroughly detailed. These measurements provided new contour minima of $\sigma_{\mathrm{p}}=4.3 \times 10^{-3} \mathrm{pb}$ at $35 \mathrm{GeV} / \mathrm{c}^{2}$ in the spindependent sector of astroparticle dark matter-proton interactions and $\sigma_{\mathrm{N}}=3.6 \times 10^{-6} \mathrm{pb}$ at $35 \mathrm{GeV} / \mathrm{c}^{2}$ in the spin-independent sector. These results were examined with respect to the fluorine spin and halo parameters used in the previous data analysis.

\section{Bubble chamber developments}

The principle challenges are a low cost $25 \mathrm{~L} \mathrm{BC}$ capable of withstanding repeated 16 bar overpressures while recording recoil events with an overall background rate of $\sim 10^{-6}$ evts $/ \mathrm{kgF} / \mathrm{d}$, and $\alpha$-recoil discrimination ability with better than $99 \%$ efficiency. The essential engineering $R \& D$, must be working hand-in-hand with the radioactive contaminants of the materials otherwise suitable for chamber construction. An example of one of the 4 prototype chambers and 2 recompression systems is seen in Fig. 1. The recompression system (Fig. 1 (left)) simultaneously controls two separate BCs ( $\sim 20 \mathrm{~L}$ volume each), with a $2 \mathrm{~s}$ response and $164 \mathrm{~s}$ overall recycling times, with both the time and pressure threshold adjustable. Larger chamber volumes require a larger substitution of the current piston.

The chambers of Fig. 1 (right) consisted of a commercially-available home water filters capable of withstanding a repeated 10 bar over-pressuring, with an estimated overall background contribution of $\sim 10^{-2}$ recoil evts/kgF/d from radio-assays. It possessed an internal $1 \mathrm{~cm}$ thick, transparent, purified gel sheathing to reduce homogeneous nucleation events [1]. The proposed chamber design concept differs from Fig. 1b, primarily by the extension of the sheathing to $\geq 10 \mathrm{~cm}$ because of the U/Th content of the commonlyavailable, inexpensive plastic containments assayed at $\sim 10^{-2}$ recoil evts $/ \mathrm{kgF} / \mathrm{d}$. It consists of a solid block of purified gel in which a cylindrical liquid containment hole is bored such that the bore hole is surrounded by a minimum $10 \mathrm{~cm}$ of gel $4 \pi$. The gel itself is contained by a low background material such as acrylic plastic, with the contribution of its U/Th contaminants 


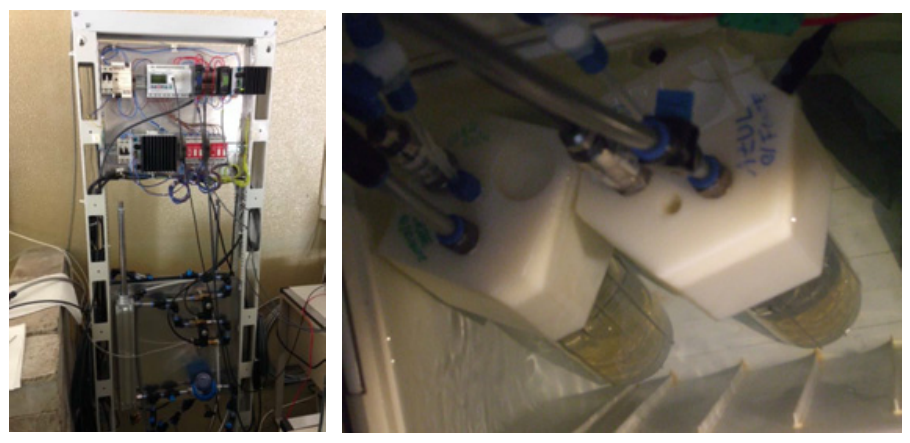

Figure 1. Left: piston-based recompression system, right: $1 \mathrm{~kg} \mathrm{C}_{2} \mathrm{ClF}_{5} \mathrm{BCs}$.

moderated by $\sim 10 \times$ by the displacement. A chamber pressure increase is easily triggered by a spontaneous nucleation event originating in imperfections in the containment surface: use of the previously-tested gel sheathing, which smoothes this interface, results in a $\sim 20 \times$ increase in time between recompressions [2].

$\mathrm{BC}$ pressure control is assured via a piston pushing or releasing high purity glycerin as necessary into the container. The halocarbons used as active liquids in our tests are immiscible in glycerin and denser, thus falling to the gel sheeted bottom of the container, leaving the glycerin to provide a smooth interface between halocarbon and detector cap. A set of electrovalves controls the system. The electrovalves are controlled via the relay outputs of a Crouzet M3 Millenium logic controller, which continuously monitors the BC pressure signal from a pressure transducer assembled on the container. The $\mathrm{BC}$ temperature is measured by means of a thermocouple type $\mathrm{k}$, inserted in the $\mathrm{BC}$ glycerin through a feedthrough in the cap.

\section{Backgrounds \& simulations}

The purified gel sheathing provides a further shielding to both the containment U/Th decays radiations and diffusion of external $\alpha$-producing radon into the chamber (which are problematic because of $(\alpha, n)$ reactions with the materials). The projected WIMP search sensitivity demands an intrinsic background contribution to the measurements of $\leq 10^{-6}$ events $/ \mathrm{kgF} / \mathrm{d}$. Previous materials' contributions, was at the level of $10^{-4}-10^{-5} \mathrm{evt} / \mathrm{kgF} / \mathrm{d}$ [3]. This level is to be reduced via a series of new materials selections based on (a) materials characteristics and (b) inherent U/Th composition. The performed radioassays of materials samples are executed by using a combination of chemical analyses of compositions, ion beam analysis of the hydrogen content of the materials (elastic recoil detection and nuclear reaction analysis + elastic backscattering, respectively), $\gamma / \alpha$ spectroscopy, and comparative neutron activation analysis. Materials requiring increased sensitivity levels, particularly in Th.

The radio-assay results are used in existing MCNP transport simulations [3] of the expected detector response with modified programs already developed. Since neutron yield and spectrum in a particular material depend not only on its radio-purity but also on its material composition and formation (e.g. the U/Th decay chains in steel are not in secular equilibrium because of the fabrication process), changing of a material (for instance, using plastic instead of PTFE or aluminium instead of stainless steel) to reduce the background rate will be investigated. Radio-assay of various plastics identified materials with a factor 10 less background; the internal sheathing can be increased to distance the containment (as well as a previously-developed spatial-resolving system implemented [4]). Recoil simulations are also 

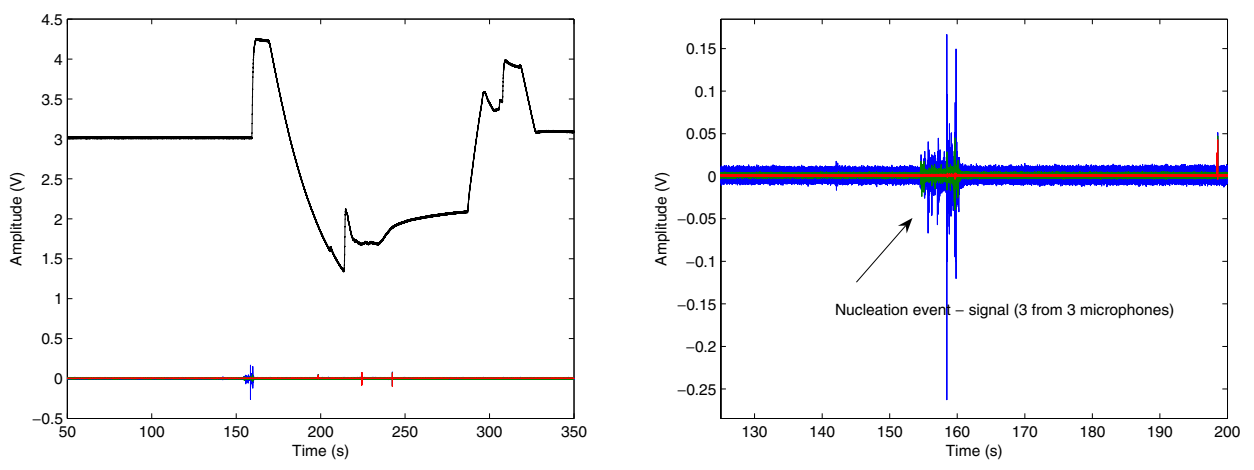

Figure 2. Left: $\mathrm{BC}$ pressure signal (top) and signal from the three microphones coupled to the PET container (bottom) during a nucleation/recompression cycle of a $\mathrm{C}_{2} \mathrm{ClF}_{5}$ filled $\mathrm{BC}$ at $9^{\circ} \mathrm{C}$, where the halocarbon was allowed to boil for some time without triggering the recompression system, right: zoomed nucleation events captured by the 3 low frequency microphones.

performed for calibration sources/configurations, to ensure: (i) the on-target source event rate exceeds background at least 10x, and (ii) a low rate of pile-up events.

\section{Nucleation event signal detection}

As recently demonstrated the sound of a gas bubble nucleation in a bubble chamber shows a broad emission up to a frequency of $250 \mathrm{kHz}$. For this reason, two different types of microphones for the SIMPLE BC prototype have been used: (a) An externally-polarized condenser microphone (CM16/CMPA40-5V from Avisoft-Bioacoustics), with a flat response over the $10-150 \mathrm{kHz}$ frequency range (high frequency microphone); (b) A PANASONIC (omnidirectional) high quality electret microphone (MCE-200), with a frequency range of $20 \mathrm{~Hz}-16 \mathrm{kHz}(3 \mathrm{~dB})$, with a signal-to-noise ratio (SNR) of $58 \mathrm{~dB}$ and a sensitivity of $7.9 \mathrm{mV} / \mathrm{Pa}$ at $1 \mathrm{kHz}$, already used with success for acoustic nucleation event detection in SDDs [5]. Several bubble chambers were assembled and deployed for testing at the Lisbon SIMPLE laboratory; one was filled with with $\sim 800 \mathrm{~g}$ of $\mathrm{C}_{2} \mathrm{ClF}_{5}$ and deployed at $9^{\circ} \mathrm{C}$ in temperature (kept constant in a water bath) and 2.0 to 3.0 bar pressure (absolute). Figure 2 shows the preliminary results of experiment using the $\mathrm{C}_{2} \mathrm{ClF}_{5}$-filled $\mathrm{BC}$ at $9^{\circ} \mathrm{C}$. One can see the $\mathrm{BC}$ pressure signal -top- and microphone signals -bottom- during an event/recompression cycle in the $\mathrm{C}_{2} \mathrm{ClF}_{5}$ filled $\mathrm{BC}$ at $9^{\circ} \mathrm{C}$ and 3 bar pressure. The signals were digitized with the NI PCI-6251 DAQ, at an acquisition rate of $300 \mathrm{kS} / \mathrm{s}$.

Preliminary frequency spectrum of the acoustic signal identified as resulting from a nucleation event. The recompression system allowed for a reliable pressure trigger from $\mathrm{BC}$ pressure differences $(\Delta \mathrm{p})$ resulting from gas bubble nucleation as low as $\Delta \mathrm{p}=100 \mathrm{mbar}$, without spurious recompression cycles. The time between the observed pressure increase and the maximum recompression pressure being reached depends on the $\mathrm{N}_{2}$ pressure and the length of the tubing connecting the piston and the BC. Typically, for a $5 \mathrm{~m} \mathrm{long}, 10 \mathrm{~mm}$ diameter tube, it is around 6 seconds. Decompression times can be regulated by means of a fine metering valve, and were typically set in the 30-60 sec interval. The noise level throughout the trials was $6.27 \pm 1.37 \mathrm{mV}$.

Analyses of the frequency microphone signals placed on the container external wall around this pressure/time stamp indicate the presence of an acoustic pulse with shape similar to a true nucleation event from a SDD. Figure 3 shows the low frequency microphones 


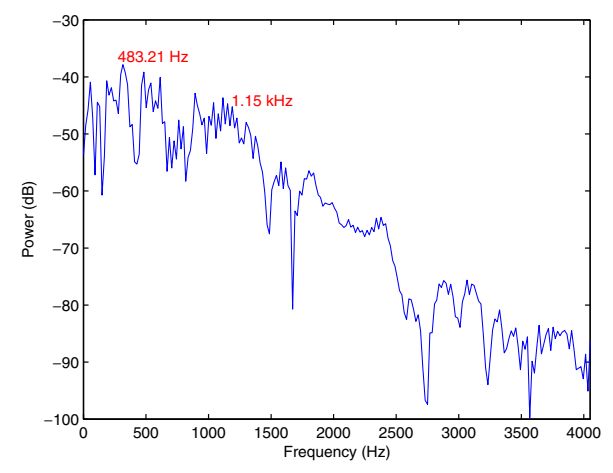

Figure 3. Frequency spectrum of the nucleation events seen in Fig. 2.

response on the container walls of the events in Fig. 2. There is a clear low frequency component with several harmonics perfectly distinguishable from other external sources of noise. Data was analysed as in the previous SIMPLE phase [5].

These preliminary results are of great importance for future BC dark matter searches since they can permit with the intrinsic background expected a clear discrimination of acoustic backgrounds from particle-induced signals with low frequency instrumentation, which has been well-demonstrated, as well as discriminations between neutron- and alpha-induced events with previous SIMPLE detectors [6-9].

\section{Future performance tests - response studies and field tests}

Following successful response testing of each BC, it will be taken to the LSBB [10] for full field, low background testing at 1500 mwe underground. The measurement site (GESA) is $60 \mathrm{~m}^{3}$, containing $\sim 8$ ton of water shielding surrounding a HDPE + wood platform supporting a temperature-controlling $400 \mathrm{~L}$ water-bath. New background event simulations are made for neutrons originating from U/Th contaminations and $(\alpha, n)$ reactions of the environment, as well as new shielding components [4]. Previous SIMPLE results have shown that the expected GESA background rate excluding its water-shielding is at the level of $10^{-5} \mathrm{evt} / \mathrm{kgF} / \mathrm{d}$. The water-bath and shield water, originally simply tap water with a background contribution of $\sim 10^{-3}$ recoil evts $/ \mathrm{kgF} / \mathrm{d}$, is to be resin-purified which reduces the heavy metals content to $2 \times 10^{-4} \mathrm{~g} / \mathrm{g}$, from which a factor 100 decrease in the current water $\mathrm{U} / \mathrm{Th}$ content to yield $\sim 10^{-5} \mathrm{evt} / \mathrm{kgF} / \mathrm{d}$ is estimated [3].

\section{Impacts}

Successful completion of the response measurements alone results in new "zero-background" 100-500 kgd exposures providing new results competitive with the current direct measurements, and comparable with both indirect and LHC results; successful project completion also results in a demonstrated new tool in the physics detectors "toolbox" for rare event search activities, which can be rapidly upgraded to larger, multi-ton arrays for deployment. With a 2-BC field test measurements, the results may contribute immediately to the question of astroparticle dark matter. The projected "zero-background" limits are shown in Fig. 4, indicating sensitivities of order $10^{-4}(\mathrm{SD}), 10^{-7}$ (SI) pb, providing further evidence for/against an astroparticle explanation for dark matter. 

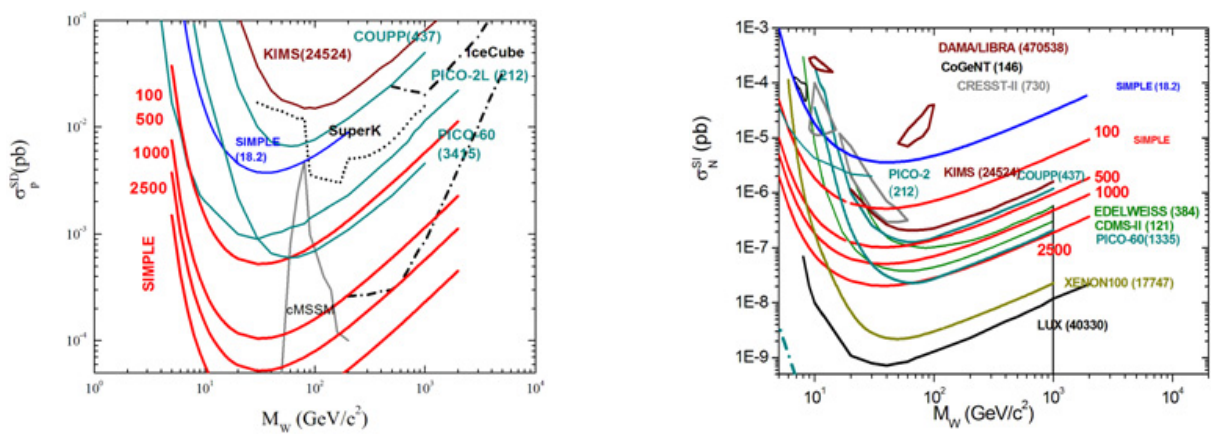

Figure 4. Left: background-free $\mathrm{S}_{\mathrm{p}} \mathrm{D}$ results with $\mathrm{C}_{2} \mathrm{ClF}_{5}$ at $6 \mathrm{keV}$ recoil detection threshold; right: same for background-free SI results.

\section{Conclusions}

Rare event searches are by nature an expensive technology arena, in terms of both their search-dictated large mass and exposure requirements. A fully functional SIMPLE prototype bubble chamber and its command and control system were developed that mitigates the initial assumptions of costs. Successful project accomplishment opens a new pathway to resolution of the question of Astroparticle dark matter existence. The anticipated results should equal or surpass the leading results. The BC technology (thermodynamic response, acoustic instrumentation) differs from others in its mechanism, opening new lines of industrial involvement.

This work is supported by $\mathrm{C}^{2}$ TN/IST and by grants PTDC/FIS/115733/2009, PTDC/FIS/121130/2010, PTDC/EEI-ELC/2468/2014 and IF/00628/2012/CP0171/CT0008. C ${ }^{2}$ TN/IST authors gratefully acknowledge the FCT support through the UID/Multi/04349/2013 project. The activity of M. Felizardo is supported by FCT grant SFRH/BPD/94028/2013.

\section{References}

[1] T.A. Girard, et al.: Proc. (iDUST -2014), Apt, France, May 5-7 (2014).

[2] J. Puibasset, PhD Thesis, Univ. Paris, (unpublished) (2000).

[3] A.C. Fernandes, et al., Nucl. Instr. \& Meth. A 623 (2010) 960.

[4] M. Felizardo, et al., Nucl. Instr. \& Meth. A 599 (2009) 93.

[5] M. Felizardo, et. al., Nucl. Instr. Meth. A 589 (2008) 72.

[6] M. Felizardo, et al., Phys. Rev. D 89 (2014) 072013.

[7] M. Felizardo, et. al., Phys. Rev. Lett. 105 (2010) 211301.

[8] M. Felizardo, et. al., Phys. Rev. Lett. 108 (2012) 201302.

[9] A.C. Fernandes, et al., Astroparticle Physics 76 (2016) 48-60.

[10] LSBB http://www. Isbb.eu 